\title{
NOUVELle
}

\section{Endocytose dans les dendrites}

\section{Un outil local de régulation de la transmission synaptique}

David Perrais ${ }^{1,2}$, Morgane Rosendale ${ }^{1-3}$
${ }^{1}$ Université of Bordeaux, 33000 Bordeaux, France.

${ }^{2}$ CNRS, UMR 5297, Institut interdisciplinaire de neurosciences, 146, rue Léo Saignat, 33077 Bordeaux, France.

${ }^{3}$ Adresse actuelle : Institut des sciences du cerveau, RIKEN, Wako, Saitama 351-0198, Japon.

david.perrais@u-bordeaux.fr

synaptiques dans le bouton présynaptique; soit le trafic membranaire est distribué sur l'ensemble de la dendrite et il ne contribue pas à la spécificité synaptique. Diverses observations permettent d'argumenter en faveur de l'un ou l'autre de ces modèles. La présence de zones d'endocytose directement dans les épines dendritiques [6] suggère en effet que leur activité pourrait être spécifiquement régulée pour moduler localement le nombre de récepteurs, qui sont présents dans une synapse stimulée (modèle 1). À l'inverse, les récepteurs peuvent eux-mêmes diffuser à la surface des neurones sur de grandes distances [7] et une modulation de leurs propriétés de diffusion pourrait donc leur permettre d'atteindre des zones d'endocytose, même éloignées, sans que les mécanismes de l'endocytose ne soient affectés (modèle 2). Pour pouvoir trancher entre ces deux scénarios, ou définir un modèle intermédiaire, il est nécessaire de visualiser avec précision les différentes étapes du trafic membranaire ou, alternativement, de pouvoir inhiber spécifiquement et localement les différentes étapes.

Pour détecter l'exocytose des vésicules contenant différents types de récepteurs, des sondes composées de la «pHluorine», une protéine naturellement fluorescente de type GFP (green fluorescent protein) sensible au $\mathrm{pH}$, fusionnée à une protéine membranaire, ont été utilisées depuis plusieurs années. La pHluorine est localisée du côté extracellulaire de la membrane plasmique (donc tournée soit vers la surface, quand le récepteur est pré- 


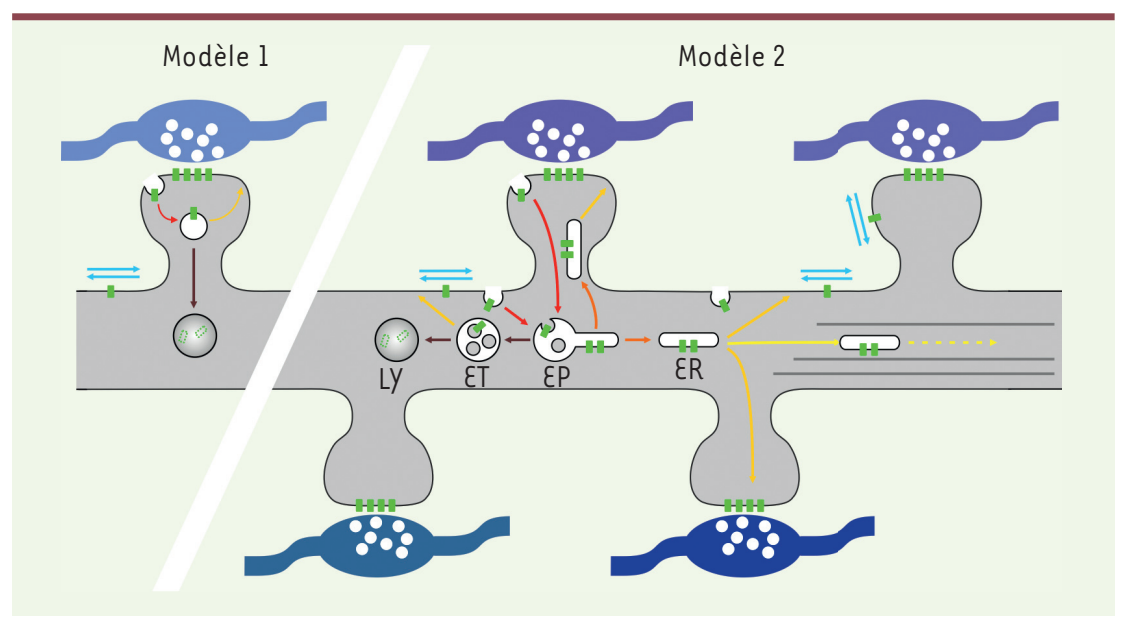

Figure 1. Deux modèles du trafic des récepteurs dans les dendrites. Les récepteurs postsynaptiques, en vert, sont concentrés dans les densités postsynaptiques (DPS), en face des boutons présynaptiques (en bleu). Les récepteurs quittent la DPS et ils diffusent dans la membrane plasmique (flèches bleu clair). Ils peuvent ensuite être concentrés dans les puits d'endocytose et internalisés (flèches rouges). Après internalisation ils sont triés dans les endosomes précoces $(\varepsilon P)$ et ils sont envoyés dans les endosomes tardifs (ET), qui maturent en lysosomes (LY) dans lesquels les récepteurs sont dégradés. Les récepteurs peuvent aussi être envoyés dans les endosomes de recyclage ( $\varepsilon R$ ), qui vont fusionner avec la membrane (flèches orange). Certaines organelles peuvent migrer rapidement sur les microtubules (flèches jaunes). Deux synapses voisines sont en général en face de neurones présynaptiques différents (différentes nuances de bleus) et elles peuvent subir des plasticités indépendamment l'une de l'autre. Le trafic des récepteurs pourrait contribuer à cette spécificité en étant restreint à une seule synapse, ou à l'inverse pour distribuer les récepteurs vers les synapses voisines.

sent sur la membrane plasmique, soit vers l'intérieur, dans les vésicules après endocytose). Elle n'est pas fluorescente à $\mathrm{pH}$ acide (inférieur à 6), elle le devient à $\mathrm{pH}$ neutre. Le $\mathrm{pH}$ des endosomes de recyclage est acide, alors que celui du milieu extracellulaire est neutre. Ainsi, lorsqu'une vésicule d'exocytose fusionne avec la membrane, son $\mathrm{pH}$ change rapidement de 5,5 à 7,4 , et des événements d'exocytose peuvent être détectés tout au long des dendrites neuronales. Après leur libération, les récepteurs diffusent rapidement dans la membrane plasmique, ou restent agrégés, selon un mécanisme que nous avons élucidé il y a quelques années [8]. Grâce à ces méthodes, différents groupes ont détecté l'exocytose de vésicules uniques et leur modulation pendant la plasticité synaptique [9]. La localisation des phénomènes d'exocytose, au regard des synapses, est cependant sujette à controverse selon les différentes études, sûr sensibles aux changements imposés, ils deviennent donc «invisibles» à $\mathrm{pH} 5,5$. En revanche, les récepteurs internalisés dans des vésicules sont insensibles aux changements rapides de $\mathrm{pH}$; ils restent donc fluorescents même quand le $\mathrm{pH}$ extracellulaire est acide. Les vésicules d'endocytose s'acidifient progressivement, ce qui se traduit par une diminution de la fluorescence de la pHluorine, mais ce laps de temps, de plusieurs dizaines de secondes, est suffisant pour détecter leur formation (Figure 2B). Ainsi, après avoir vérifié que ce protocole n'affecte pas les neurones, nous avons pu dresser une carte des lieux de formation des vésicules d'endocytose par rapport aux synapses. Ceci nous a permis de montrer que des récepteurs non synaptiques, comme le récepteur de la transferrine, étaient internalisés dans toutes les zones d'endocytose, mais sans préférence détectable pour les zones proches ou lointaines des synapses (Figure 2C). En revanche, les récepteurs synaptiques de type AMPA ( $\alpha$-amino3-hydroxy-5-methyl-4-isoxazolepropionic acid), activés par le glutamate, sont internalisés préférentiellement, mais pas exclusivement, dans des zones d'endocytose proches des synapses (Figure 2D). Enfin, pour répondre à la question initiale, la stimulation de l'endocytose des récepteurs AMPA, dans un protocole de dépression synaptique à long terme (DLT), augmente significativement la fréquence de formation de vésicules, mais les cartes de localisation montrent que l'on perd la préférence pour les zones d'endocytose proches des synapses. Ainsi, l'activité des zones d'endocytose proches des synapses n'est pas spécifiquement stimulée pendant une DLT, ce qui suggère que l'endocytose ne contribue pas à la spécificité de la plasticité synaptique.

Ces résultats montrent qu'une localisation précise des zones d'endocytose proches des synapses suffit à internaliser préférentiellement les récepteurs synaptiques in situ. Ils confirment, de plus, que l'endocytose est importante pour la 


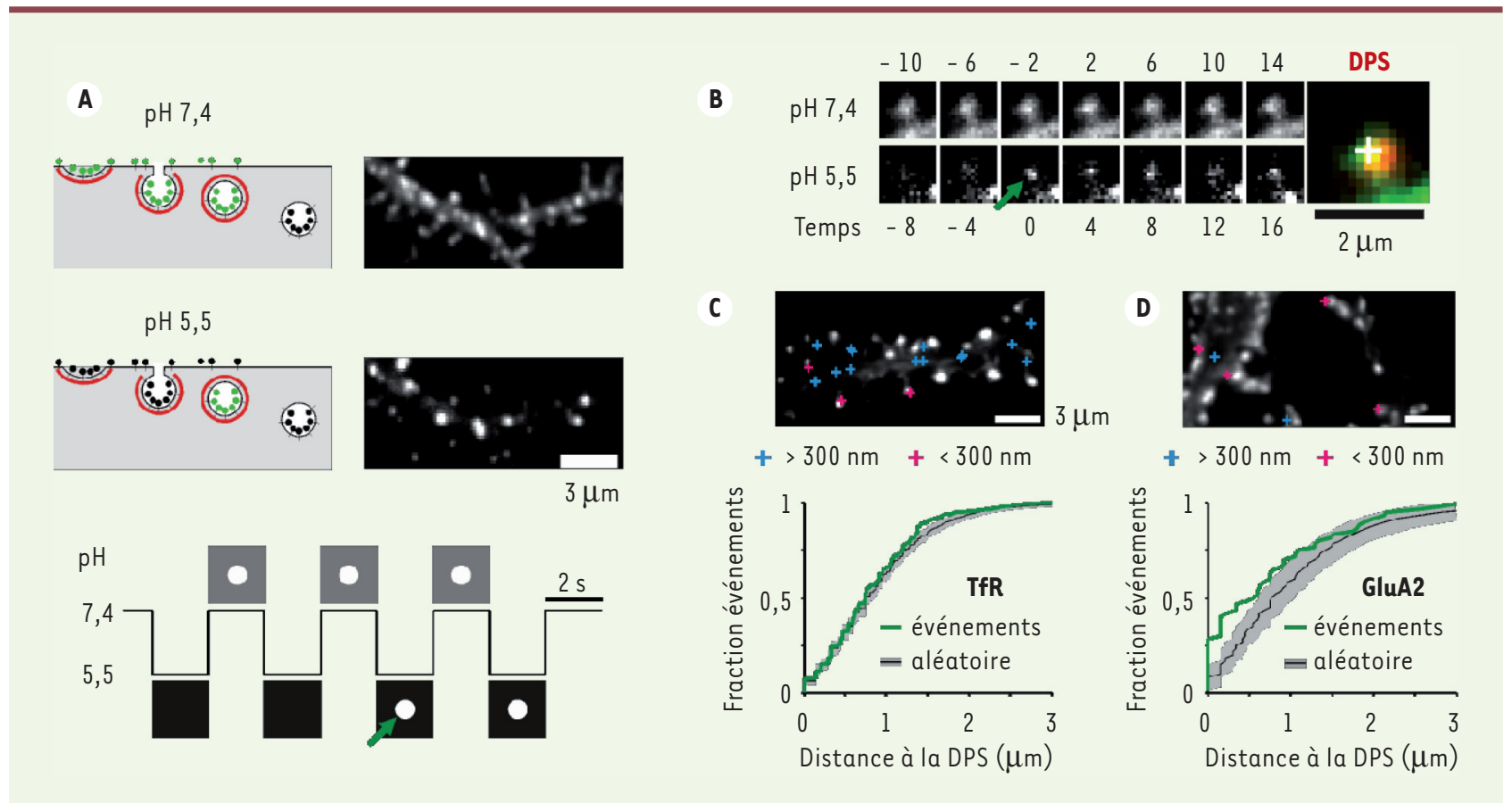

Figure 2. Visualisation de l'endocytose des récepteurs marqués par la pHluorine. A. illustration du protocole. Les neurones expriment des récepteurs marqués grâce à la pHluorine. À un pH extracellulaire de 7,4, les récepteurs présents sur la membrane plasmique et dans des vésicules non acides sont fluorescents. À un pH extracellulaire de 5,5, uniquement les récepteurs dans les vésicules non acides sont visibles. En changeant le $\mathrm{pH}$ toutes les deux secondes on peut détecter la formation de nouvelles vésicules d'endocytose (flèche verte). B. images successives d'une épine dendritique à pH 7,4 (images du haut) et 5,5 (images du bas). Au temps 0 , une vésicule apparaît, signe d'une endocytose. (flèche verte). À droite, superposition de l'image au temps 0 (vert) et de l'image d'un marqueur des DPS, Homer (rouge). C. carte des événements d'endocytose détectés comme en B pour le récepteur de la transferrine (TfR), non synaptique. En bas, la distribution des distances entre les densités postsynaptiques (DPS) et les événements d'endocytose (courbe verte) montre que ces derniers ne se concentrent pas vers les DPS, car cette distribution est superposée à une distribution d'événements répartis aléatoirement sur la surface dendritique (la courbe noire et les zones grisées représentent la médiane et les intervalles $5 \%-95 \%$ de 200 répartitions aléatoires). $D$. carte des événements d'endocytose pour le récepteur synaptique au glutamate GluA2. L'analyse des distances effectuée comme pour C montre que les événements d'endocytose sont enrichis vers les DPS (d'après [9]).

régulation de la DLT tout en suggérant qu'elle ne contribue pas directement à la spécificité synaptique. II est ainsi essentiel que les récepteurs AMPA soient internalisés au cours de la DLT, mais la machinerie de l'endocytose elle-même n'est pas localement régulée. Quels autres facteurs pourraient alors expliquer cette spécificité ? L'endocytose n'est pas l'unique étape du trafic membranaire qui régule la transmission synaptique. L'augmentation d'endocytose des récepteurs AMPA, que nous avons observée, dure moins de dix minutes alors que la dépression des synapses perdure plus longtemps. Les étapes ultérieures de l'endocytose, comme le mouvement des endosomes dans la dendrite, notamment grâce aux moteurs moléculaires, ou le trafic des récepteurs vers les endosomes tardifs, qui empêche leur recyclage vers la membrane, pourraient être également modulées (Figure 1). Pour étudier avec précision ces étapes, il est nécessaire de suivre les récepteurs, après l'acidification des vésicules d'endocytose, avec de nouvelles sondes fluorescentes. De plus, dans cette étude, l'application généralisée d'agoniste a induit l'activation de tous les récepteurs stimulant l'endocytose, contrairement à une stimulation physiologique où seuls les récepteurs d'un petit nombre de synapses sont stimulés. Dans la mesure où nous avons montré que la visualisation directe de la formation de vésicules uniques était possible, nous pourrons utiliser des protocoles plus précis pour décrire la régulation locale de l'endocytose. Toutefois, cette étude illustre comment la description détaillée des étapes du trafic membranaire et leurs modulations peut permettre de mieux comprendre comment les neurones organisent les différentes connexions synaptiques et régulent indépendamment leur plasticité. L'altération de ces processus, qui intervient probablement chez les sujets ayant des prédispositions génétiques aux maladies neurodégénératives [12, 13], pourrait compromettre le trafic de nombreux récepteurs mais également la capacité des neurones à ségréger les différentes entrées synaptiques, qui sont essentielles pour l'activité neuronale. Endocytosis in dendrites: a local tool to regulate synaptic transmission 


\section{LIENS D'INTÉRÊT}

Les auteurs déclarent n'avoir aucun lien d'intérêt concernant les données publiées dans cet article.

\section{RÉFÉRENCES}

1. Watanabe S, Rost BR, Camacho-Pérez M, et al. Ultrafast endocytosis at mouse hippocampal synapses. Nature 2013 ; 504 : 242-7.

2. Soykan T, Maritzen T, Haucke V. Modes and mechanisms of synaptic vesicle recycling. Curr Opin Neurobiol 2016 ; 39 : 17-23.

3. Choquet D, Lounis B. Mobilité des récepteurs du glutamate. Un nouveau mécanisme de contrôle de la transmission synaptique rapide dans le cerveau. Med Sci (Paris) $2008 ; 24: 548-50$.
4. Lüscher C, Xia H, Beattie $\varepsilon C$, et al. Role of AMPA receptor cycling in synaptic transmission and plasticity. Neuron $1999 ; 24: 649-58$.

5. Park M, Penick EC, Edwards JG, et al. Recycling endosomes supply AMPA receptors for LTP. Science $2004 ; 305$ : 1972-5.

6. Lu J, Helton TD, Blanpied TA, et al. Postsynaptic positioning of endocytic zones and AMPA receptor cycling by physical coupling of dynamin-3 to Homer. Neuron 2007 ; 55 : 874-89.

7. Choquet $D$, Triller $A$. The dynamic synapse. Neuron $2013 ; 80: 691-703$

8. Jullié $D$, Choquet $D$, Perrais $D$. Recycling endosomes undergo rapid closure of a fusion pore on exocytosis in neuronal dendrites. J Neurosci 2014 ; 34 : 11106-18.

9. Kennedy MJ, Davison IG, Robinson CG, Ehlers MD. Syntaxin-4 defines a domain for activity- dependent exocytosis in dendritic spines. Cell 2010 ; $141: 524-35$.

10. Rosendale M, Jullié D, Choquet D, Perrais D. Spatial and temporal regulation of receptor endocytosis in neuronal dendrites revealed by imaging of single vesicle formation. Cell Rep 2017 ; 18 : 1840-7.

11. Merrifield CJ, Perrais D, Zenisek D. Coupling between clathrin-coated-pit invagination, cortactin recruitment, and membrane scission observed in live cells. Cell 2005; 121 : 593-606.

12. Schreij AMA, Fon EA, McPherson PS. Endocytic membrane trafficking and neurodegenerative disease. Cell Mol Life Sci 2016 ; 73 : 1529-45.

13. Small SA, Petsko GA. Retromer in Alzheimer disease, Parkinson disease and other neurological disorders. Nat Rev Neurosci 2015 ; 16 : 126-132.

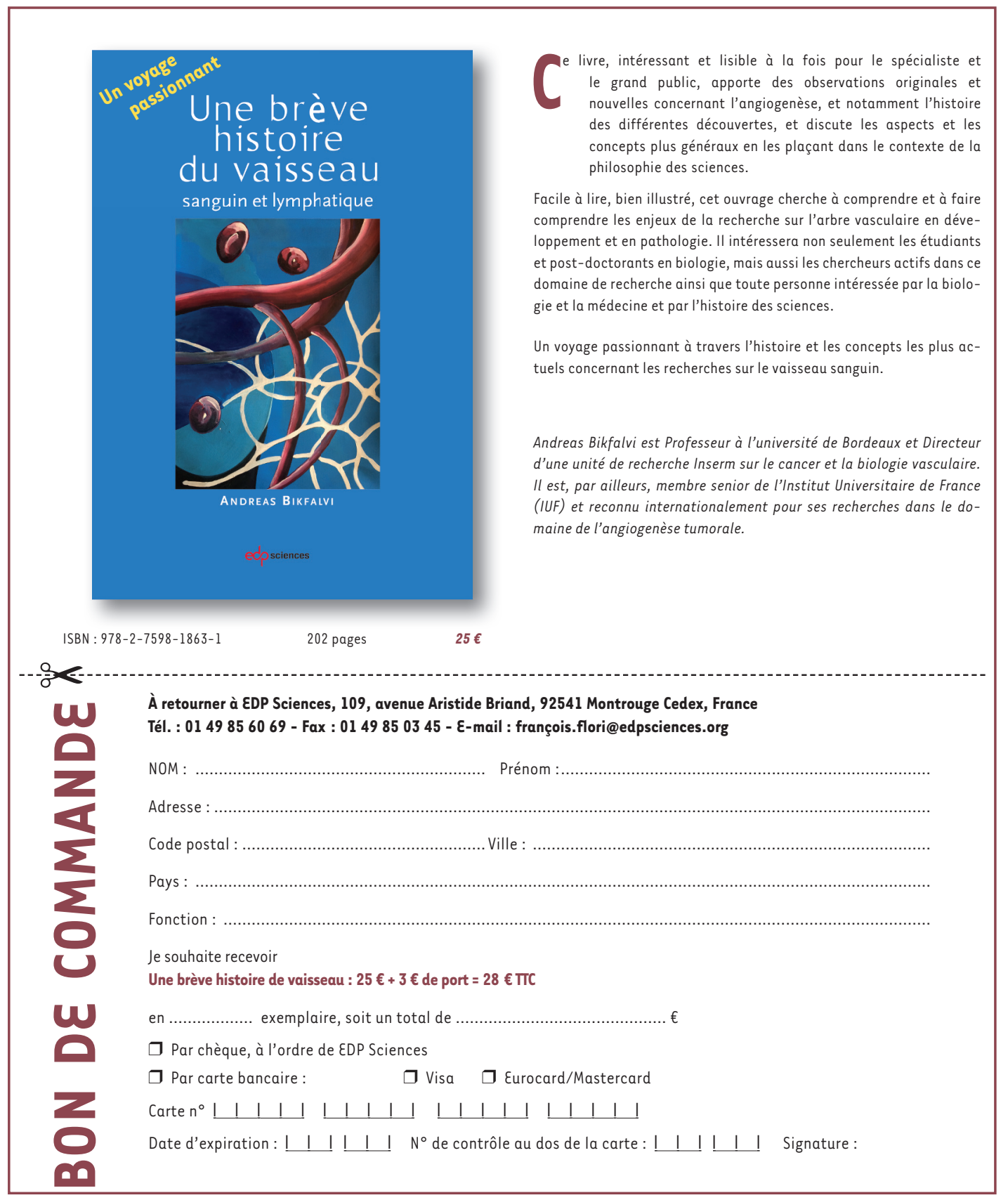

